\title{
Chronic myelogenous leukemia in the age of imatinib: assessing response, acceleration, and blast phase
}

\author{
Robert P. Hasserjian
}

Received: 5 May 2011 /Accepted: 6 May 2011 /Published online: 21 May 2011

(C) Springer-Verlag 2011

\begin{abstract}
Chronic myelogenous leukemia, $B C R-A B L 1+$ $(\mathrm{CML})$ is a myeloproliferative neoplasm defined by the presence of the $B C R-A B L$ fusion gene and a $\mathrm{t}(9 ; 22)(\mathrm{q} 34$; q11) cytogenetic abnormality. Its natural history is that of inexorable progression to an acute leukemia (blast crisis) after a prolonged chronic phase. The recent development of imatinib and other inhibitors of $B C R-A B L$ tyrosine kinase activity has dramatically altered the clinical course of CML, with long-term remissions in most patients treated early in the course of the disease. Monitoring of resistance to therapy and progression on therapy are critical in the current management of the disease. Problems facing the diagnostic pathologist in this "Age of Imatinib" include accurate diagnosis and determination of CML disease phase prior to therapy, evaluating response to therapy, and assessing for disease progression on therapy.
\end{abstract}

Keywords Chronic myelogenous leukemia $\cdot$ Philadelphia chromosome $\cdot B C R-A B L \cdot$ Imatinib $\cdot$ Tyrosine kinase inhibitors $\cdot$ Myeloproliferative neoplasms

\section{Background}

Chronic myelogenous leukemia, $B C R-A B L 1+(\mathrm{CML})$ is a myeloproliferative neoplasm defined by the presence of the

R. P. Hasserjian

Department of Pathology, Massachusetts General Hospital,

55 Fruit Street, WRN244,

Boston, MA 02114, USA

R. P. Hasserjian $(\square)$

Harvard Medical School, Massachusetts General Hospital,

55 Fruit Street, WRN244,

Boston, MA 02114, USA

e-mail: rhasserjian@partners.org
$B C R-A B L$ fusion gene that is usually associated with a $t$ $(9 ; 22)(\mathrm{q} 34 ; \mathrm{q} 11)$ cytogenetic abnormality (the Philadelphia chromosome). This fusion of the $A B L$ tyrosine kinase gene with the breakpoint cluster region gene ( $B C R$, encoding a serine-threonine kinase) produces a constitutively active chimeric protein that promotes neoplastic transformation via dysregulated phosphorylation of various substrates and activates the RAS, PI3K, and JAK-STAT pathways, among others $[1,2]$. In the vast majority of CML cases, the $B C R$ breakpoints occur in the relatively small $(5.8 \mathrm{~kb})$ major breakpoint cluster region [3] and result in a 210-kDa fusion protein (p210). Rare CML cases (about $0.3 \%$ ) have a breakpoint in the minor breakpoint cluster region, resulting in a p190 fusion protein [4]. The p190 fusion protein is far more common in Philadelphia chromosome-positive B acute lymphoblastic leukemia $(\mathrm{Ph}+\mathrm{B}-\mathrm{ALL})$ than in $\mathrm{CML}$ [5]. In very rare CML cases, the breakpoint occurs at the microbreakpoint cluster region, generating a p230 fusion protein.

The characteristic features of CML hematopoiesis include a differentiation pattern that favors the granulocytic lineage over the erythroid lineage, deregulated proliferation, growth factor independence, defective adhesion to bone marrow stromal cells, and defective apoptosis. This abnormal hematopoiesis results in bone marrow hypercellularity and the inappropriate entry of immature myeloid cells into the peripheral blood (Fig. 1a) and their colonization of extramedullary tissues such as the spleen $[6,7]$. The main clinical manifestations are leukocytosis with increased circulating immature granulocytic precursors, basophilia, and splenomegaly (Table 1). Patients typically present with clinical symptoms related to the increased white cell mass, including fatigue, weight loss, and/or abdominal discomfort due to splenomegaly, but the disease is discovered incidentally in asymptomatic patients in up to $50 \%$ of cases $[8,9]$. When untreated, the natural history of CML is that of inexorable progression to an acute leukemia (blast 
Fig. 1 Typical findings of CML in chronic phase at diagnosis. a Peripheral blood smear shows marked leukocytosis with a spectrum of maturing granulocytic elements and basophilia. b The bone marrow trephine biopsy is markedly hypercellular with an increased myeloid/erythroid ratio and numerous band and segmented granulocytes; megakaryocytes are small with simplified nuclear contours. $\mathbf{c}$ The bone marrow aspirate shows a spectrum of nondysplastic maturing granulocytic elements and increased basophils. d Karyotype of blood or bone marrow typically shows a $\mathrm{t}(9 ; 22)$ (q34;q11) abnormality (arrows)

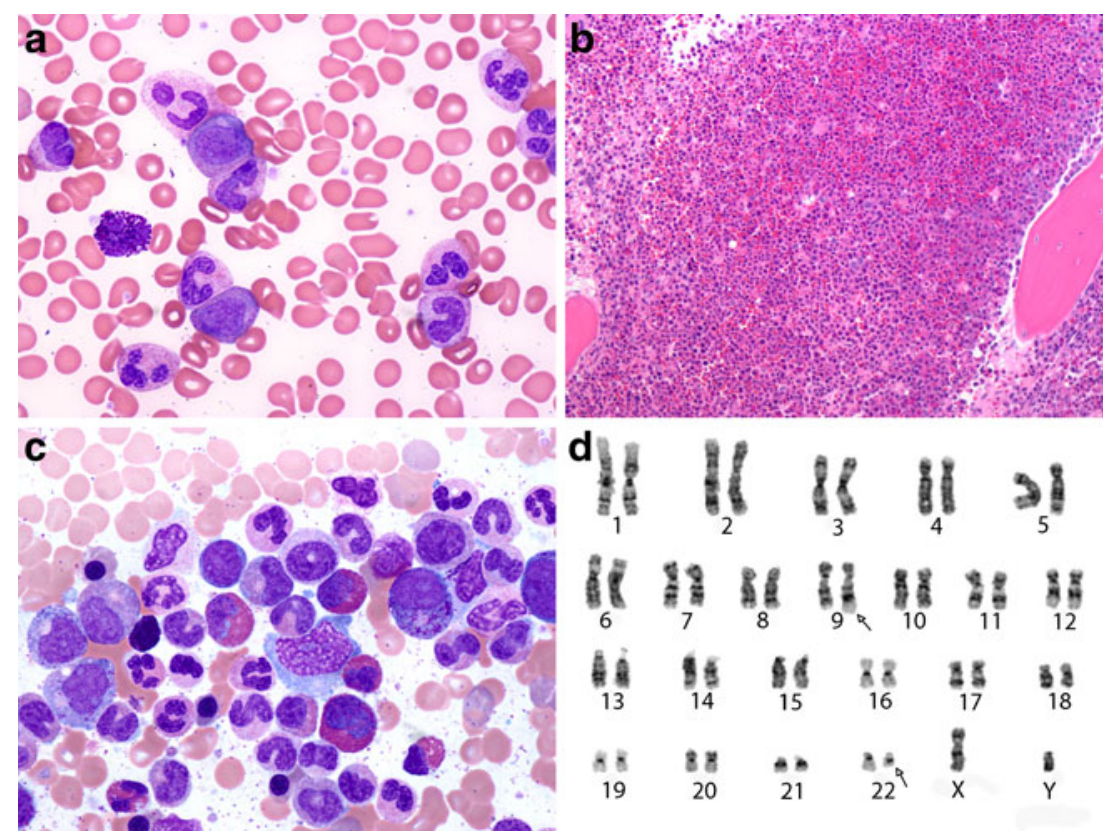

crisis) after a prolonged chronic phase. Earlier therapies used to treat CML, mainly hydroxyurea and interferon, were generally ineffective at prolonging survival or delaying disease progression in most patients. However, the development of pharmacologic inhibitors of BCR-ABL (the tyrosine kinase inhibitors (TKIs) imatinib, dasatinib, nilotinib, and bosutinib) has dramatically improved the survival of CML patients since the beginning of the twenty-first century and has radically altered the disease course.

\section{The first step: accurate diagnosis of CML is still critical in the imatinib era}

The bone marrow biopsy in CML patients at diagnosis is markedly hypercellular, usually approaching $100 \%$, with a markedly elevated myeloid/erythroid ratio (Table 1; Fig. 1b). Megakaryocytes are often increased and are usually smaller than normal, with hypolobated nuclei, a feature that distinguishes them from the typical megakaryocyte morphology of other myeloproliferative neoplasms such as essential thrombocythemia and primary myelofibrosis. This is an important distinction, because the bone marrow in CML may exhibit varying degrees of reticulin fibrosis either at diagnosis or upon progression, potentially mimicking primary myelofibrosis. The bone marrow aspirate also shows an elevated myeloid/erythroid ratio with a spectrum of all stages of granulocytic maturation represented and increased basophils and eosinophils (Fig. 1c). Unless in an advanced phase of the disease, blasts comprise $<10 \%$ of the marrow and peripheral blood cells and there is no significant morphologic dysplasia in erythroid and myeloid cells. The $B C R-A B L$ translocation can be readily detected in peripheral blood by cytogenetics, fluorescence in situ hybridization (FISH), and/or reverse transcription PCR (RT-PCR) and thus a diagnosis of CML usually can be presumptively made prior to a bone marrow biopsy [10] (Fig. 1d). However, a pre-therapy bone marrow sample is desirable for the Bone

Table 1 Typical features of chronic myelogenous leukemia in chronic phase

\begin{tabular}{|c|c|c|}
\hline Peripheral blood findings & Bone marrow findings & Cytogenetic and molecular genetic findings \\
\hline Leukocytosis (median, $100 \times 10^{9} / \mathrm{L}$ ) & Hypercellularity (approaching 100\%) & $\begin{array}{l}\text { Karyotype: } \mathrm{t}(9 ; 22)(\mathrm{q} 34: \mathrm{q} 11) \text { translocation or } \\
\text { complex translocation involving } 9 \mathrm{q} 34 \text { and } 22 \mathrm{q} 11 \text { loci }\end{array}$ \\
\hline Increased immature myeloid precursors & $\begin{array}{l}\text { Increased myeloid/erythroid ratio } \\
\text { (usually at least } 10: 1 \text { ) }\end{array}$ & FISH: fusion of $B C R$ and $A B L 1$ loci \\
\hline Eosinophilia & Increased myelocytes & RT-PCR: fusion transcript of BCR and ABL1 \\
\hline Basophilia & Basophilia & \\
\hline \multirow[t]{2}{*}{ Often thrombocytosis } & Small, hypolobated megakaryocytes & \\
\hline & $\begin{array}{l}\text { Lack of morphologic dysplasia of erythroid } \\
\text { and myeloid elements }\end{array}$ & \\
\hline
\end{tabular}


marrow blast count is an important determinant of CML phase and may be higher than in the peripheral blood. (2) Adverse cytogenetic markers of clonal evolution are more readily detected in bone marrow than in peripheral blood samples. (3) The bone marrow biopsy may show reticulin fibrosis, providing both prognostic information as well as a baseline to measure the reduction in reticulin fibrosis that occurs in most patients responding to TKIs [11]. A pre-therapy bone marrow examination, including biopsy (with reticulin stain), aspiration, and cytogenetics, is thus recommended in all newly diagnosed CML cases. Flow cytometry has no current role in the diagnosis of CML in chronic phase, but is useful in determining the blast phenotype (lymphoid, myeloid, or biphenotypic) in blast crisis (see below).

Importantly for both the diagnostic pathologist and hematologist, CML may present with atypical features that can lead to its misdiagnosis as other myeloproliferative neoplasms. Given the highly effective targeted therapies available to treat CML, molecular genetic testing should be performed to exclude CML in any suspected myeloproliferative process. The absence of $B C R-A B L$ effectively excludes CML. However, in cases that are highly suspicious for CML and are unexpectedly negative for $B C R-A B L$ by a single testing modality (cytogenetics, FISH, or RT-PCR), an additional test should be performed to exclude the possibility of occasional false negative results that can occur with any of these tests; for example, about $5 \%$ of cases of CML cases lack a classic t $(9 ; 22)$ due to a variant translocation or a cryptic $B C R-A B L$ fusion. Long-template qualitative RT-PCR can detect most variants and thus represents a highly sensitive method of detecting $B C R-A B L$ in putative CML cases at diagnosis [12, 13]. However, some variant translocations may escape detection by even long-template RT-PCR [14-16]. The pathologist should be aware of this possibility and request confirmatory multiplex PCR testing and/or FISH in rare cases of CML that are falsely negative by RT-PCR [17].

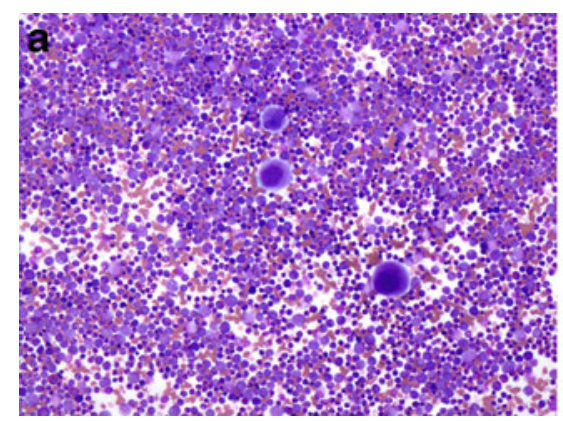

Fig. 2 CML presenting with unusual features mimicking other neoplasms. a Patient with moderate leukocytosis and absolute monocytosis (absolute monocyte count $5.6 \times 10^{9} / \mathrm{L}$ ); although the marked myeloid hyperplasia and small, hypolobated megakaryocytes in the bone marrow aspirate suggest CML, RT-PCR failed to detect BCR-ABL translocation. Cytogenetics showed a $t(9 ; 22)$ and repeat RT-PCR using different primers revealed a variant e1a2 BCR-ABL
Atypical presentations of CML that can challenge the diagnostician include cases with blunted leukocytosis due to concomitant metabolic deficiencies, marked eosinophilia, prominent thrombocytosis that may be more impressive than the leukocytosis, or monocytosis. These atypical presentations can mimic other myeloid neoplasms, leading to a missed diagnosis of CML and a missed opportunity to provide effective TKI therapy. CML cases with an increased absolute monocyte count can be mistaken for chronic myelomonocytic leukemia (CMML). This is an important distinction, as CMML (as defined by the WHO 2008 Classification) does not respond to TKI therapy. Monocytes only rarely comprise $>10 \%$ of all peripheral blood leukocytes in CML, and cases with $>10 \%$ monocytes are often associated with a rare p190 (e1a2) BCR-ABL (Fig. 2a, b). Atypical CML, BCR$A B L$ negative (often referred to in earlier publications as "Philadelphia-negative CML") also presents with leukocytosis and immature myeloid elements, but lacks basophilia and often has dysplastic morphology. Chronic neutrophilic leukemia $(\mathrm{CNL})$ is a rare myeloproliferative neoplasm that also presents with leukocytosis, but lacks significant circulating immature granulocytic elements or basophilia. CML patients with p230 BCR-ABL may present with a mature neutrophilia resembling CNL or with prominent thrombocytosis mimicking essential thrombocythemia [18]. (Fig. 2c, d). Most importantly, atypical CML, $B C R$ $A B L$ negative, CMML, and CNL all lack $B C R-A B L$ by definition; thus molecular genetic testing is the most reliable method to distinguish these entities from CML. The pathologist must be astute to these atypical presentations and should suggest $B C L-A B L$ testing if $C M L$ is even a remote possibility.

Rarely, CML patients may present in blast crisis (CML$\mathrm{BC})$, mimicking a de novo Ph+ ALL or acute myeloid leukemia (AML). In contrast to $\mathrm{Ph}+\mathrm{ALL}$ that usually bears the e1a2 p190 BCR-ABL variant, only very rare cases of

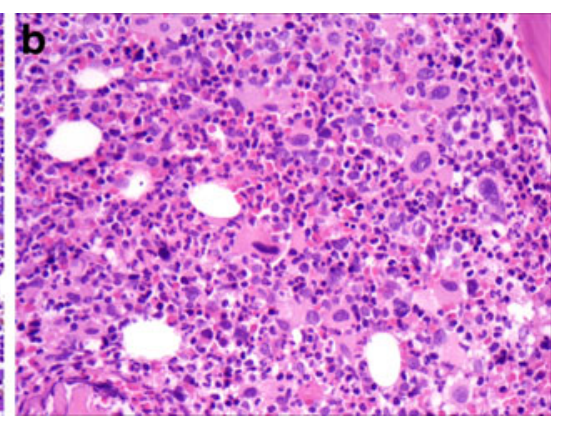

fusion (p190 protein). b Patient presenting with a platelet count of $1,093 \times 10^{9} / \mathrm{L}$ and only minimal leukocytosis. The bone marrow trephine is markedly hypercellular with small, hypolobated megakaryocytes unlike the enlarged megakaryocytes with complex nuclear lobation seen in essential thrombocythemia. Cytogenetics revealed a $t$ $(9 ; 22)$, establishing the diagnosis of CML 
CML show the e1a2 variant [4]. Distinguishing rare cases of de novo $\mathrm{Ph}+\mathrm{AML}$ from CML-BC may be more difficult, as p210 BCR-ABL characterizes both. Unlike CML-BC, $\mathrm{Ph}+\mathrm{AML}$ usually lacks basophilia and is less likely to manifest with massive splenomegaly [19]. Cases of presumed acute leukemia treated with induction chemotherapy in which the post-induction bone marrow shows no acute leukemia, but morphologically resembles chronic phase of disease (CML-CP) and continues to show a $t$ $(9 ; 22)$ by conventional cytogenetics, likely represent cases of CML presenting in blast crisis.

\section{Prognosis of CML at diagnosis}

The natural history of CML is characterized by evolution through three clinical phases of disease. Patients usually present in the chronic phase of disease (CML-CP), characterized by indolent and clinically stable features. However, about $10 \%$ of CML patients present in advanced phases, termed accelerated phase (CML-AP) or blast crisis (CML-BC), without a clinically evident chronic phase [20]. Even with the advent of TKI therapy, the phase of disease remains an important prognostic factor in CML [21, 22]. According to the 2008 WHO Classification, bone marrow and peripheral blood blast count, peripheral blood basophil and platelet counts, unresponsiveness to cytoreductive therapy, and cytogenetics are the parameters used to define CML-AP [10] (see Table 2). One recent study suggests that imatinib-treated CML patients with $20-29 \%$ blasts (currently classified as CML-BC according to WHO Classification criteria) have a prognosis similar to CML-AP rather than other CML-BC patients [23]. Although not a defining feature of CML-AP in the WHO Classification, marked reticulin and/or collagen fibrosis in the bone marrow accompanied by a prominent increase in megakaryocytes is also associated with disease progression, and is used as a defining feature of CML-AP according to International Bone Marrow Transplant Registry criteria [24].

Aside from disease phase, several other clinicopathologic parameters have been identified at the time of diagnosis that correlate with time to progression and survival. The Sokal score incorporates age spleen size, platelet count, and blast percentage in the peripheral blood, which are combined into a hazard ratio. The Sokal scoring system was developed at the time of earlier CML therapies, but it retains its prognostic significance even in TKI-treated CML patients [25]. Increased bone marrow reticulin fibrosis represents an independent adverse prognostic risk factor in interferon and hydroxyurea-treated CML patients [26] and pre-treatment increased reticulin has also been associated with a lower rate of cytogenetic remission in imatinib-treated CML patients [27].

While the development of additional chromosomal abnormalities represents progression to at least CML-AP, the significance of chromosomal abnormalities additional to $t(9 ; 22)$ at the time of diagnosis is uncertain. Additional chromosomal abnormalities present at diagnosis were associated with a poor prognosis in earlier studies, but not in more recent studies examining imatinib-treated patients [28-30]. Gene expression profiling shows promise in risk-stratifying CML patients treated with TKI. In one study, microarray analysis of $\mathrm{CD} 34+$ progenitor cells in CML-CP cases revealed differential gene expression in patients with indolent disease from those who rapidly progressed to CML-BC; specifically, lower expression of CD7 and higher expression of proteinase 3 or elastase were associated with a more indolent course [31]. CD7 expression in CD34+ CML blasts detected by flow cytometry has also been independently associated with adverse prognosis in CML-CP [32, 33]. Several studies have also shown differential gene expression between peripheral blood leukocytes from CML cases that subsequently respond or do not respond to imatinib [34-36].

Table 2 WHO classification 2008 staging system for chronic myelogenous leukemia [10]

\begin{tabular}{|c|c|c|c|}
\hline Feature & Chronic phase & Accelerated phase & Blast crisis \\
\hline Blast $\%$ in blood or bone marrow & $<10 \%$ & $10-19 \%$ & $\geq 20 \%$ \\
\hline Basophil \% in blood & $<20 \%$ & $\geq 20 \%$ & NA \\
\hline Platelet count $\left(\times 10^{9} / \mathrm{L}\right)$ & $\begin{array}{l}\leq 1,000 \text { or responsive to therapy if }>1,000 \\
\geq 100 \text { or related to therapy if }<100\end{array}$ & $\begin{array}{l}>1,000 \text {, unresponsive to therapy } \\
<100 \text {, unrelated to therapy }\end{array}$ & NA \\
\hline White blood count $\left(\times 10^{9} / \mathrm{L}\right)$ & $\leq 10$ or responsive to therapy if $>10$ & $>10$, unresponsive to therapy & NA \\
\hline Splenomegaly & Responsive to therapy & $\begin{array}{l}\text { Persistent or increasing, } \\
\text { unresponsive to therapy }\end{array}$ & NA \\
\hline Extramedullary blast tumor (chloroma) & Absent & Absent & Present \\
\hline New cytogenetic changes after initial karyotype ${ }^{a}$ & Absent & Present & NA \\
\hline
\end{tabular}

NA not applicable

${ }^{a}$ Most frequently observed changes of cytogenetic evolution are additional Philadelphia chromosome, isochromosome 17q, trisomy 8 , and/or trisomy 19 
One recent study showed differential expression of a single drug metabolism gene, PTGS1/COX1, in CML cases that had primary resistance to TKI therapy; in contrast, the signature of secondary TKI resistance that did not involve the common $B C R-A B L \mathrm{KD}$ mutations was more complex, including up to 11 different genes [37]. These studies suggest that gene expression profiling in the future may obviate the current "trial-and-error" practice of using molecular monitoring (see below) to guide the use of TKI versus alternative therapies for CML.

\section{Evaluating response to therapy}

Imatinib therapy is highly effective in treating CML, but its effectiveness is limited in some patients by the development of disease resistance. Primary resistance to TKIs is infrequent and is usually related to drug-efflux mechanisms or $B C R-A B L$ amplification that overcome the inhibitory effects of the drug [38, 39]. Secondary resistance, occurring after an initial response to TKI, is more common and is frequently due to mutations in the $B C R-A B L$ kinase domain (KD) that abrogate the inhibitory activity of imatinib and/or other TKIs [40]. These KD mutations develop as a result of selection pressure and correlate with progression to advanced phases and shortened survival. Thus, early identification of imatinib resistance is critical to allow the use of other, potentially effective therapies, such as secondgeneration TKIs and/or stem cell transplantation [41]. Cytogenetic analysis and molecular genetic testing to quantify the disease burden are the cornerstones of disease monitoring of CML patients on TKI therapy.

The clinical and genetic definitions of responses to TKI therapy are shown in Table 3. Imatinib and other
TKIs induce a complete hematologic remission (CHR; defined by normalization of blood counts and spleen size) in nearly all patients treated in chronic phase. CHR is usually accompanied by normalization of bone marrow cellularity, myeloid/erythroid ratio, megakaryocyte number and morphology, and reduction of any reticulin fibrosis that was present prior to therapy [11] (Fig. 3a). These changes are accompanied by increased apoptosis of CML cells and are maintained with long-term imatinib therapy, provided that a cytogenetic response persists [42]. Cytogenetic responses are measured by the percentage of at least 20 bone marrow metaphases that bear the $\mathrm{t}(9 ; 22)$ translocation $(\mathrm{Ph}+$ metaphases): a major cytogenetic response (MCR) represents $\leq 35 \%(<7 / 20) \mathrm{Ph}+$ metaphases, with a partial response being $1-35 \% \mathrm{Ph}+$ metaphases and a complete response (CCR) being $0 \% \mathrm{Ph}+$ metaphases. CML patients showing cytogenetic response to imatinib will almost always exhibit CHR and morphologic normalization of bone marrow appearance. However, in the first year of TKI therapy, complete hematologic responses and morphologic normalization of marrow may occur even in patients lacking a cytogenetic response, underscoring the need to monitor residual disease using genetic and/or molecular genetic techniques [11] (Fig. 3b). However, a lack of cytogenetic response may be suspected on the basis of bone marrow morphology if small megakaryocytes are prevalent, patches of increased reticulin fibrosis persist, or bone marrow basophilia is present [43] (Fig. 3c-f). Indeed, small megakaryocytes present in CML that persists on imatinib therapy have been shown to be $B C R-A B L$ positive by FISH analysis, indicating their derivation from the persistent CML clone [44].

Most imatinib-treated CML-CP patients achieve CCR and failure to achieve at least a MCR is associated with
Table 3 Definitions of response of CML on imatinib and therapeutic goals $[46,49,50,86]$

\footnotetext{
${ }^{\mathrm{a}}$ Failure to meet expected therapeutic targets indicates a suboptimal response, and should elicit TKI-resistance mutation analysis and/or switch of therapy

${ }^{\mathrm{b}} \mathrm{BCR}-\mathrm{ABL}$ transcript ratio $=$ ratio of $\mathrm{BCR}-\mathrm{ABL}$ transcript level to transcript level of a reference gene, as assessed by real-time quantitative RT-PCR (RQ-PCR)
}

Hematologic parameters: CHR expected within 3 months of imatinib therapy ${ }^{\mathrm{a}}$

CHR Complete hematologic response White blood count of $<10 \times 10^{9} / \mathrm{L}$, basophils of $<5 \%$, no myeloid precursors in blood, platelets of $<450 \times 10^{9} / \mathrm{L}$, and normalization of spleen size

Cytogenetic parameters: at least a minor CR expected within 3 months, MCR expected within 6 months, and CCR expected within 12 months of imatinib therapy ${ }^{\mathrm{a}}$

Minor CR Minor cytogenetic response $\quad 36-65 \% \mathrm{Ph}+$ metaphases on bone marrow cytogenetics

MCR $\quad$ Major cytogenetic response $\quad \leq 35 \% \mathrm{Ph}+$ metaphases on bone marrow cytogenetics

CCR Complete cytogenetic response $\quad 0 \% \mathrm{Ph}+$ metaphases (at least 20 counted) on bone marrow cytogenetics

Molecular genetic parameters (RQ-PCR): MMR expected within 18 months of therapy ${ }^{\mathrm{a}}$

MMR Major molecular response $\quad \geq 3-\log$ reduction in BCR-ABL transcript ratio ${ }^{\mathrm{b}}$ in blood compared with pre-treatment reference level by RQ-PCR assay

CMR Complete molecular response Negativity for BCR-ABL by sensitive RQ-PCR assay 
Fig. 3 Morphologic features of CML treated with TKIs. a Mildly hypercellular bone marrow trephine in a 66-year-old woman treated with imatinib for 1 year: megakaryocytes and myeloid/ erythroid ratio appear normal and cytogenetics shows complete cytogenetic remission. b Similarappearing bone marrow trephine in a 61-year-old woman treated with dasatinib for 6 months; however, cytogenetics reveals persistent $\mathrm{t}(9 ; 22)$ in all 20 metaphases, indicating treatment failure with discordant "normalization" of bone marrow appearance. c Reticulin staining of bone marrow trephine from (b) shows focal fiber increase, which can be a clue to persistent disease. $\mathbf{d}$ Even in normocellular marrow, frequent hypolobated megakaryocytes in the bone marrow trephine also correlate with treatment failure or relapse. e, f Small hypolobated megakaryocytes with rounded nuclei can also be seen in the aspirate smears

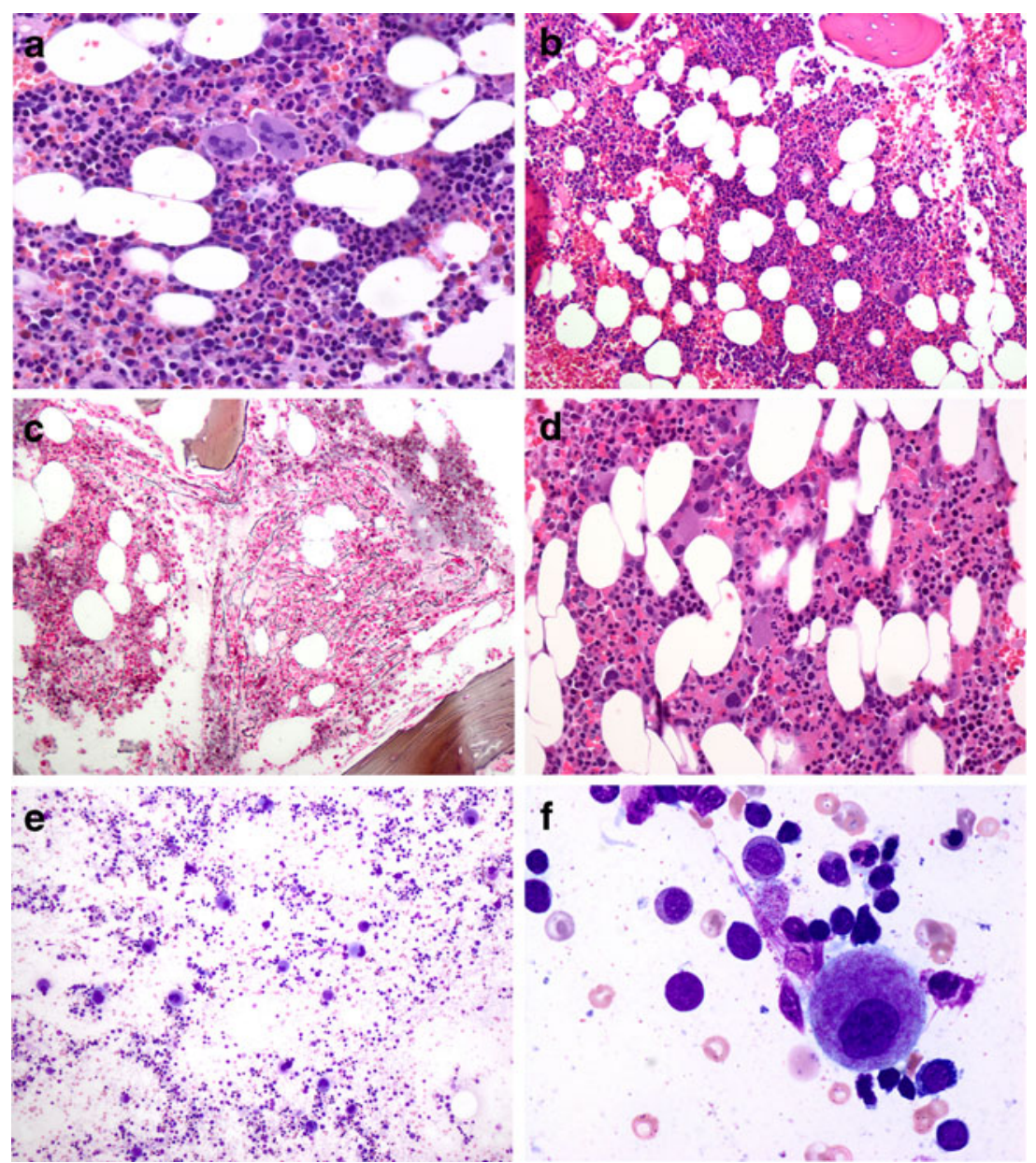

increased risk of progression to advanced phases of disease and shortened survival [45]. Expected responses for newer TKIs (nilotinib and dasatinib) are more rapid than for imatinib [46]. Although cytogenetic analysis of bone marrow is considered to be the "gold standard" of cytogenetic responsiveness, interphase FISH analysis of peripheral blood to detect $B C R-A B L$ appears to correlate well with cytogenetic responsiveness and is used in lieu of bone marrow cytogenetic analysis at some centers [47]. However, periodic bone marrow cytogenetic analysis to confirm CCR and to assess for clonal evolution is still indicated during disease monitoring on therapy $[48,49]$.

While the achievement of a CCR is a desirable goal with imatinib therapy, real-time quantitative RT-PCR (RQ-PCR) currently represents the "gold standard" to detect and quantify low levels of persistent CML. Molecular response by RQ-PCR is defined as the detectable BCR-ABL transcript ratio (ratio of the $\mathrm{BCR}-\mathrm{ABL}$ transcript level to the level of a reference gene transcript, usually either BCR, $\mathrm{ABL}$, or GUSB) as a percentage of the average BCR-ABL transcript ratio of a control group of pre-therapy $\mathrm{CML}$ cases, established at each laboratory [50]. BCR-ABL transcript levels can be determined on blood or bone marrow. Although a quantitative technique, RQ-PCR results may be variable between and within laboratories [51]. Ideally, RQ-PCR analyses on a given patient should be done consistently on the same type of sample (usually blood) and in the same laboratory over the course of his/her treatment [51]. Major molecular response (MMR) represents a decrease in the BCR-ABL transcript ratio of at least 3 logs from the baseline (i.e., $0.1 \%$ of the transcript level present at initial diagnosis). Achieving MMR is the goal of imatinib therapy and is achieved in $50-60 \%$ of CML patients by 12 months of therapy and in up to $80 \%$ of patients after 4 years of therapy [1]. Patients achieving MMR have excellent progression-free survival with continued imatinib therapy and rapid attainment of MMR is particularly associated with long-term responsiveness to imatinib [52]. Surprisingly, low-level detectable BCR-ABL transcripts persist in the vast majority of CML patients with MMR, in spite of continued imatinib therapy and long-term disease free progression and only $10-40 \%$ of patients achieve a complete molecular response (CMR),which is defined as undetectable BCR-ABL transcript in a RQ-PCR assay that has at least $4-5 \log$ dynamic range $[25,48]$. The reasons for this persistent low-level disease are uncertain; 
however, the durability of MMR in most patients indicates that imatinib can keep a small residual CML population in a quiescent state with low likelihood of relapse and very low incidence of progression to advanced phase disease. Importantly, even CMR cannot be equated to "cure," as $61 \%$ of imatinib-treated CML patients achieving a CMR for at least 2 years relapsed after stopping imatinib therapy; most relapses occurred rapidly, within 6 months of stopping therapy [53].

Although there is some variability in practice, most current centers monitor disease by RQ-PCR in blood every 3 months during the first 12 months of TKI therapy, then every 6 months once CCR is attained [49]. Bone marrow cytogenetic studies should be performed prior to initiating TKI therapy and every 6 to 12 months until CCR, then every 1-2 years. These frequent periodic cytogenetic and molecular response assessments are performed in order to reveal patients that respond suboptimally to imatinib therapy (see Table 3). Suboptimal response, defined by a failure to achieve specific cytogenetic and/or molecular response targets at the designated timepoints in Table 3, correlates with resistance to imatinib and warrants an investigation for KD mutations and/or a switch to another therapy. Loss of CCR or hematologic relapse in a previously responding patient should also elicit resistance mutation analysis. A sustained or increasing rise in transcript level of 2- to 5-fold even at very low disease levels has been associated with an increased likelihood of detecting TKI-resistance mutations [54, 55], but most centers use a 10-fold increase in transcript levels above MMR to trigger mutation analysis or to consider a change in therapy [56]. Frequent monitoring by the above methods is important, since CML failing initial TKI therapy is more likely to respond to a second-line therapy if the therapeutic switch is instituted rapidly [57].

The most common mechanism of secondary resistance to TKI is mutation in the KD of $B C R-A B L . \mathrm{KD}$ mutations can be detected by several methods, including pyrosequencing, mutation-specific RQ-PCR, liquid bead arrays, and denaturing high performance liquid chromatography, but direct sequencing is by far the most common [58]. Although it is the least sensitive method, direct sequencing is the most routinely available and reliable method to cover all possible mutation sites and has been recommended for KD mutation analysis by an international consensus panel [59]. Mutations that confer TKI-resistance cluster in four critical regions in the $B C R-A B L \mathrm{KD}$, involving ATP binding (P-loop), imatinib binding, catalytic activity, and kinase activation (A-loop). Mutations vary in their ability to inhibit the currently available TKIs and thus the specific mutation type can help guide the use of a TKI that is not inhibited by that particular mutation. The mutation at amino acid 315 in the imatinib-binding site (T315I mutation) confers resistance to imatinib, dasatnib, and nilotinib by preventing access of these drugs to the ATP-binding pocket.

The mechanisms by which these mutations develop are poorly understood. KD mutations can be present at low-levels in CML cases prior to exposure to TKI therapy. However, these small mutant clones do not necessarily confer an adverse prognosis and resistant clones may not expand even under the selection pressure of TKI therapy [60,61]. For these reasons, up-front assessment for KD mutations is not recommended prior to the institution of TKI therapy, as it may not predict resistance to TKI therapy. Secondary resistance to TKIs due to KD mutations usually occurs within the first 2 years of TKI therapy and is much more common in CML-AP and CML-BC than CML-CP [25]. Although KD mutations are a common cause of secondary resistance to TKI therapy, about half of the resistance occurrences are due to other mechanisms [21]. These include BCR-ABL overexpression (due to amplification at the genomic level or other mechanisms) and rare mutations outside the KD. Mechanisms of resistance not directly involving $B C R-A B L$ are often associated with cytogenetic evolution that results in the activation of other oncogenes and/or loss of tumor suppression genes. Gene expression profiling may be helpful in identifying target genes associated with KDmutation-independent TKI resistance [37]. Alteration in drug metabolic kinetics is usually a mechanism of primary TKI resistance to TKI and only rarely a cause of secondary TKI resistance.

\section{Determining progression on therapy}

In the absence of effective therapy, CML patients inexorably progress to blast crisis. This blast crisis may exhibit a myeloid (about $60 \%$ of cases), lymphoid (20-30\% of cases), or mixed myeloid-lymphoid phenotype (10-25\% of cases), and may manifest in extramedullary organs as a myeloid sarcoma [62-64]. The median time to progression from CML-CP to CML-BC in untreated patients or in patients treated with older therapies such as hydroxyurea ranges from 3 to 5 years [65]. Blast crisis is typically preceded by an intermediate accelerated phase of disease (CML-AP) by a period of 4-6 months [66], but up to $25 \%$ of CML-BC cases develop without any clinically evident preceding accelerated phase [67]. Disease progression is uncommon currently in CML patients treated in chronic phase with TKIs. When it occurs, progression usually occurs early during treatment: in one series, $3.3 \%$ of CML$\mathrm{CP}$ patients progressed to CML-AP/CML-BC within the first 18 months of therapy, while $<1 \%$ progressed after 4 years of therapy [68]. Rare cases may abruptly progress to 
Fig. 4 Progression of CML on TKI therapy. a Progression to myeloid blast crisis after 6 months of imatinib therapy: the bone marrow trephine cellularity is replaced by sheets of myeloid blasts interspersed with small megakaryocytes typical of CML. b Rapid development of lymphoid blast crisis after only 4 months of imatinib therapy, with sheets of small lymphoid blasts predominating in the bone marrow aspirate smear. c, $\mathbf{d} \mathrm{Pa}$ tient treated in chronic phase with imatinib but tolerated therapy poorly and developed splenic rupture after 2 months. Splenectomy specimen shows red pulp replacement by sheets of myeloid blasts, representing extramedullary myeloid blast crisis. e Patient treated with imatinib, but not monitored and presented after 2 years with pancytopenia. Bone marrow trephine is markedly fibrotic with focal sheets of blasts (upper right, adjacent to bone); $30 \%$ myeloid blasts were present in the aspirate smear (not shown), confirming myeloid blast crisis. f CML in blast crisis with numerous blasts and prominent immature basophils
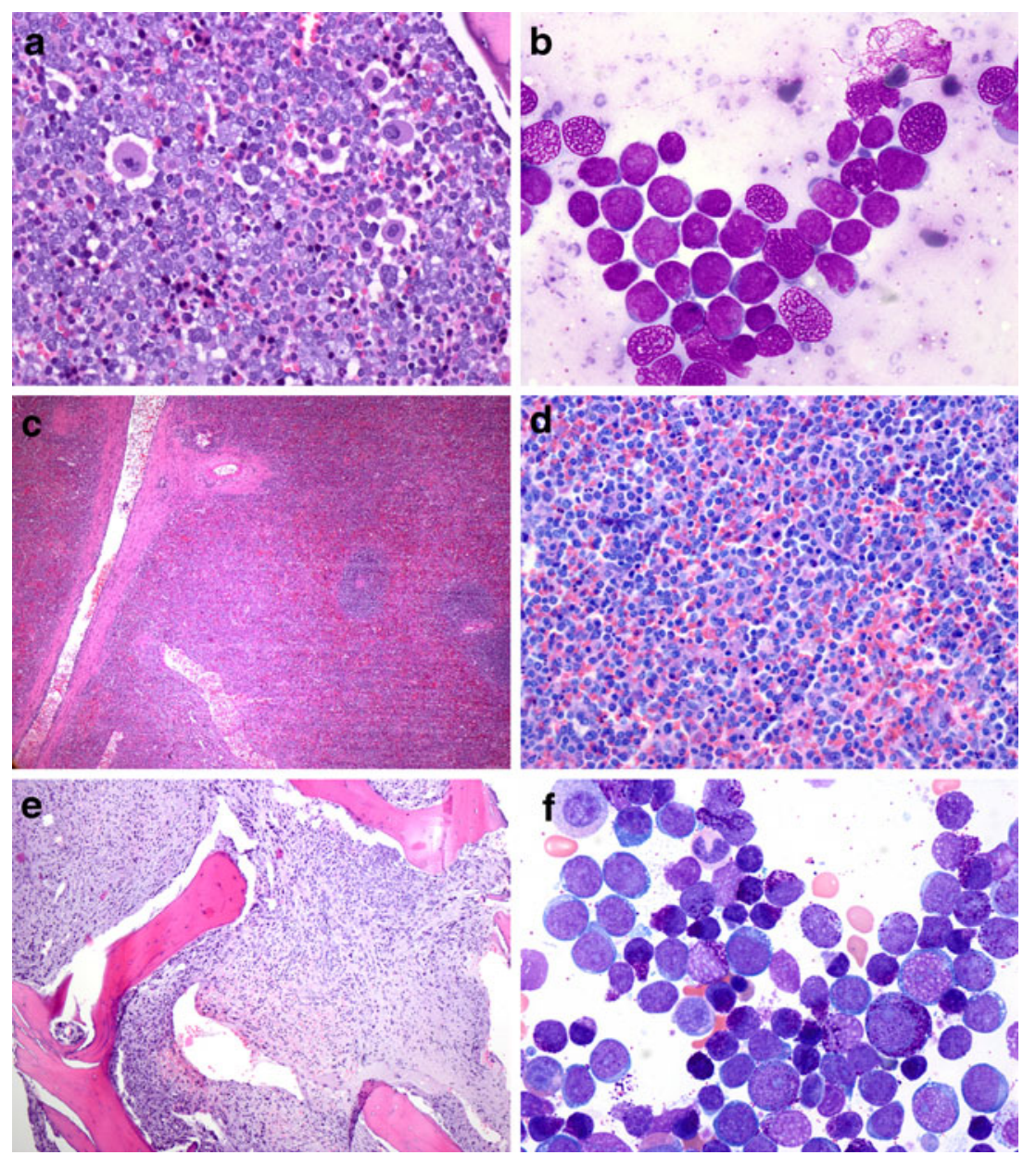

blast crisis, although this is uncommon if rigorous clinical, cytogenetic, and molecular monitoring is performed [69] (Fig. 4). In particular, central nervous system relapse has been reported to occur as in CML patients treated with imatinib and diagnosticians must be vigilant to this presentation $[70,71]$.
Fig. 5 Development of cytogenetic abnormalities in non-CML cells during TKI therapy. a A 27year-old woman with complete molecular response to imatinib developed pancytopenia with a hypocellular bone marrow trephine. b The bone marrow aspirate shows left-shifted myeloid maturation with abnormal retention of primary granules and dyserythropoiesis. c Abnormal pseudo-Pelger-Huet granulocytes are seen in the peripheral blood. d Conventional cytogenetics showed monosomy 7 in 18 of 20 metaphases and no $t(9 ; 22)$. Unlike most patients developing new non-CML cytogenetic abnormalities on TKI therapy, this patient developed a bona fide myelodysplastic syndrome and required a bone marrow transplant

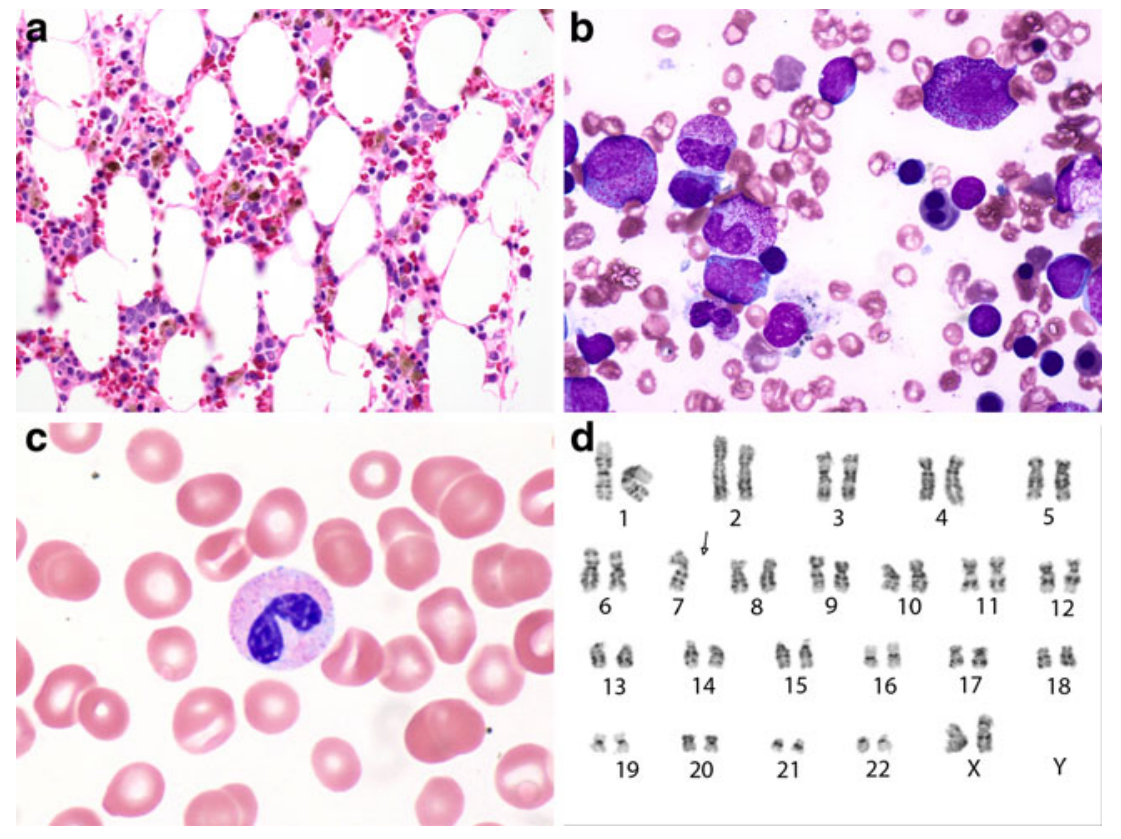


The progression to advanced phases of disease is caused by the development of genetic lesions in addition to $B C R$ $A B L$; secondary cytogenetic changes are identified in $85 \%$ of patients by the time of progression to CML-BC [72]. Even in the absence of other features of CML-AP or CML$\mathrm{BC}$, the acquisition of new cytogenetic abnormalities in patients treated with imatinib is associated with loss of response and poor prognosis [30, 73]. FISH appears to increase the yield of identifying the most common additional chromosomal abnormalities associated with disease progression, but it is unclear if these small clones with secondary abnormalities have clinical relevance [74]. Thus, the use of FISH to examine for additional secondary changes that are not evident on routine karyotype is not currently indicated.

The most frequent secondary cytogenetic changes in advanced phase CML are trisomy 8 , one or more additional Philadelphia chromosomes (derivative 22), and an isochromosome 17 [75]. The molecular events associated with CML progression are complex and are likely related to genetic instability and impaired DNA repair mechanisms that are consequences of BCR-ABL activity [76-78]. In addition to cytogenetic aberrations, $B C R-A B L$ amplification, gene mutations, and epigenetic changes such as gene methylation also appear to play a role in disease progression [79]. Interestingly, different blast crisis phenotypes have been be associated with unique patterns of molecular evolution: $A B L$ kinase domain mutations that confer resistance to TKIs occur frequently in lymphoid blast crisis, while myeloid blast crisis is more commonly associated with cytogenetic evolution. $B C R-A B L$ amplification is seen in about one third of both myeloid and lymphoid blast crisis cases [4]. The criteria for CML-AP in patients progressing on TKI therapy are still poorly defined and disease progression in CML is currently defined more practically in terms of responsiveness to TKI therapy [50]. Evidence of therapeutic refractoriness (such as rising RQ-PCR BCR-ABL levels or loss of CCR) should elicit a bone marrow examination [80]. However, even if standard features of CML-AP or CML-BC are not pathologically evident, a switch in therapy may be indicated in such cases. Of note, increased reticulin fibrosis on imatinib therapy is independently associated with treatment failure and appears more significant than increased reticulin present at the initial diagnosis. For these reasons, it is important to perform a reticulin stain on CML bone marrow samples taken on TKI therapy [27].

\section{Other pathologic processes occurring in CML patients treated with tyrosine kinase inhibitors}

While therapeutic refractoriness to imatinib is often associated with cytogenetic evolution, between $2 \%$ and
$8 \%$ of CML patients treated with imatinib develop clonal cytogenetic abnormalities in non-CML cells lacking a $t(9 ; 22)$ [81, 82]. The abnormalities include changes often associated with myelodysplastic syndromes, such as trisomy 8 (the most frequently observed), deletion $7 \mathrm{q}$, deletion $20 \mathrm{q}$, and loss of the $\mathrm{Y}$ chromosome. Although often associated with cytopenias, these changes are usually transient and only rare patients develop myelodysplastic syndromes [83] (Fig. 5). The etiology of these cytogenetic abnormalities is uncertain: normal hematoopoiesis following successful TKI therapy has been shown to be polyclonal in most patients and imatinib does not appear to be mutagenic in vitro $[84,85]$. Further follow-up is required to determine the incidence and significance of these cytogenetic changes. Until their significance and followup are clarified, bone marrow examination is recommended in CML patients on TKI therapies who develop new onset unexplained cytopenias [49].

Conflicts of interest The author declares that he has no conflict of interest.

\section{References}

1. Druker BJ, O'Brien SG, Cortes J, Radich J (2002) Chronic myelogenous leukemia. Hematology Am Soc Hematol Educ Program 111-135

2. Ren R (2005) Mechanisms of BCR-ABL in the pathogenesis of chronic myelogenous leukaemia. Nat Rev Cancer 5:172-183

3. Melo JV (1996) The diversity of BCR-ABL fusion proteins and their relationship to leukemia phenotype. Blood 88:23752384

4. Jones D, Luthra R, Cortes J et al (2008) BCR-ABL fusion transcript types and levels and their interaction with secondary genetic changes in determining the phenotype of Philadelphia chromosome-positive leukemias. Blood 112:5190-5192

5. Lugo TG, Pendergast AM, Muller AJ, Witte ON (1990) Tyrosine kinase activity and transformation potency of bcr-abl oncogene products. Science 247:1079-1082

6. Gordon MY, Dowding CR, Riley GP, Goldman JM, Greaves MF (1987) Altered adhesive interactions with marrow stroma of haematopoietic progenitor cells in chronic myeloid leukaemia. Nature 328:342-344

7. Goldman JM, Melo JV (2003) Chronic myeloid leukemiaadvances in biology and new approaches to treatment. N Engl J Med 349:1451-1464

8. Faderl S, Talpaz M, Estrov Z, O’Brien S, Kurzrock R, Kantarjian HM (1999) The biology of chronic myeloid leukemia. N Engl J Med 341:164-172

9. Kantarjian HM, Smith TL, McCredie KB et al (1985) Chronic myelogenous leukemia: a multivariate analysis of the associations of patient characteristics and therapy with survival. Blood 66:1326-1335

10. Vardiman J, Melo J, Baccarani M, Thiele J (2008) WHO classification of tumours of haematopoietic and lymphoid tissues. International Angency for Research on Cancer (IARC), Lyon, pp $32-37$ 
11. Hasserjian RP, Boecklin F, Parker S et al (2002) ST1571 (imatinib mesylate) reduces bone marrow cellularity and normalizes morphologic features irrespective of cytogenetic response. Am J Clin Pathol 117:360-367

12. Luthra R, Sanchez-Vega B, Medeiros LJ (2004) TaqMan RT-PCR assay coupled with capillary electrophoresis for quantification and identification of bcr-abl transcript type. Mod Pathol 17:96-103

13. Branford S, Hughes $T$ (2006) Diagnosis and monitoring of chronic myeloid leukemia by qualitative and quantitative RTPCR. Methods Mol Med 125:69-92

14. Burmeister T, Reinhardt R (2008) A multiplex PCR for improved detection of typical and atypical BCR-ABL fusion transcripts. Leuk Res 32:579-585

15. Schultheis B, Wang L, Clark RE, Melo JV (2003) BCR-ABL with an e6a2 fusion in a CML patient diagnosed in blast crisis. Leukemia 17:2054-2055

16. Hochhaus A, Reiter A, Skladny H et al (1996) A novel BCR-ABL fusion gene (e6a2) in a patient with Philadelphia chromosomenegative chronic myelogenous leukemia. Blood 88:2236-2240

17. Dessars B, El Housni H, Lambert F, Kentos A, Heimann P (2006) Rational use of the EAC real-time quantitative PCR protocol in chronic myelogenous leukemia: report of three false-negative cases at diagnosis. Leukemia 20:886-888

18. Pane F, Frigeri F, Sindona M et al (1996) Neutrophilic-chronic myeloid leukemia: a distinct disease with a specific molecular marker (BCR/ABL with C3/A2 junction). Blood 88:2410-2414

19. Soupir CP, Vergilio JA, Dal Cin P, Muzikansky A, Kantarjian H, Jones D, Hasserjian RP (2007) Philadelphia chromosome-positive acute myeloid leukemia: a rare aggressive leukemia with clinicopathologic features distinct from chronic myeloid leukemia in myeloid blast crisis. Am J Clin Pathol 127:642-650

20. Quintas-Cardama A, Cortes JE (2006) Chronic myeloid leukemia: diagnosis and treatment. Mayo Clin Proc 81:973-988

21. Jabbour E, Kantarjian H, Jones D et al (2006) Frequency and clinical significance of BCR-ABL mutations in patients with chronic myeloid leukemia treated with imatinib mesylate. Leukemia 20:1767-1773

22. Mitchell B, Deininger M (2011) Techniques for risk stratification of newly diagnosed patients with chronic myeloid leukemia. Leuk Lymphoma 52(Suppl 1):4-11

23. Cortes JE, Talpaz M, O'Brien S et al (2006) Staging of chronic myeloid leukemia in the imatinib era: an evaluation of the World Health Organization proposal. Cancer 106:1306-1315

24. Speck B, Bortin MM, Champlin R, Goldman JM, Herzig RH, McGlave PB, Messner HA, Weiner RS, Rimm AA (1984) Allogeneic bone-marrow transplantation for chronic myelogenous leukaemia. Lancet 1:665-668

25. Druker BJ, Guilhot F, O'Brien SG et al (2006) Five-year followup of patients receiving imatinib for chronic myeloid leukemia. $\mathrm{N}$ Engl J Med 355:2408-2417

26. Buesche G, Hehlmann R, Hecker H et al (2003) Marrow fibrosis, indicator of therapy failure in chronic myeloid leukemiaprospective long-term results from a randomized-controlled trial. Leukemia 17:2444-2453

27. Buesche G, Ganser A, Schlegelberger B, von Neuhoff N, Gadzicki D, Hecker H, Bock O, Frye B, Kreipe H (2007) Marrow fibrosis and its relevance during imatinib treatment of chronic myeloid leukemia. Leukemia 21:2420-2427

28. Sokal JE, Gomez GA, Baccarani M et al (1988) Prognostic significance of additional cytogenetic abnormalities at diagnosis of Philadelphia chromosome-positive chronic granulocytic leukemia. Blood 72:294-298

29. Farag SS, Ruppert AS, Mrozek K et al (2004) Prognostic significance of additional cytogenetic abnormalities in newly diagnosed patients with Philadelphia chromosome-positive chronic myelogenous leukemia treated with interferon-alpha: a Cancer and Leukemia Group B study. Int J Oncol 25:143-151

30. Cortes JE, Talpaz M, Giles F et al (2003) Prognostic significance of cytogenetic clonal evolution in patients with chronic myelogenous leukemia on imatinib mesylate therapy. Blood 101:3794 3800

31. Yong AS, Szydlo RM, Goldman JM, Apperley JF, Melo JV (2006) Molecular profiling of CD34+ cells identifies low expression of CD7, along with high expression of proteinase 3 or elastase, as predictors of longer survival in patients with CML. Blood 107:205-212

32. Martin-Henao GA, Quiroga R, Sureda A, Garcia J (1999) CD7 expression on $\mathrm{CD} 34+$ cells from chronic myeloid leukaemia in chronic phase. Am J Hematol 61:178-186

33. Kosugi N, Ebihara Y, Nakahata T, Saisho H, Asano S, Tojo A (2005) CD34+CD7+ leukemic progenitor cells may be involved in maintenance and clonal evolution of chronic myeloid leukemia. Clin Cancer Res 11:505-511

34. Kaneta Y, Kagami Y, Katagiri T et al (2002) Prediction of sensitivity to STI571 among chronic myeloid leukemia patients by genome-wide cDNA microarray analysis. Jpn J Cancer Res 93:849-856

35. Ohno R, Nakamura Y (2003) Prediction of response to imatinib by cDNA microarray analysis. Semin Hematol 40:42-49

36. McLean LA, Gathmann I, Capdeville R, Polymeropoulos MH, Dressman M (2004) Pharmacogenomic analysis of cytogenetic response in chronic myeloid leukemia patients treated with imatinib. Clin Cancer Res 10:155-165

37. Zhang WWCJ, Yao H, Zhang L, Reddy NG, Jabbour E, Kantarjian HM, Jones D (2009) Predictors of primary imatinib resistance in chronic myeloid leukemia are distinct from those in secondary imatinib resistance. J Clin Oncol 27:3642-3649

38. Dulucq S, Bouchet S, Turcq B, Lippert E, Etienne G, Reiffers J, Molimard M, Krajinovic M, Mahon FX (2008) Multidrug resistance gene (MDR1) polymorphisms are associated with major molecular responses to standard-dose imatinib in chronic myeloid leukemia. Blood 112:2024-2027

39. White DL, Saunders VA, Dang P et al (2007) Most CML patients who have a suboptimal response to imatinib have low OCT-1 activity: higher doses of imatinib may overcome the negative impact of low OCT-1 activity. Blood 110:4064-4072

40. Branford S, Rudzki Z, Walsh S, Grigg A, Arthur C, Taylor K, Herrmann R, Lynch KP, Hughes TP (2002) High frequency of point mutations clustered within the adenosine triphosphatebinding region of $\mathrm{BCR} / \mathrm{ABL}$ in patients with chronic myeloid leukemia or $\mathrm{Ph}$-positive acute lymphoblastic leukemia who develop imatinib (STI571) resistance. Blood 99:3472-3475

41. Soverini S, Martinelli G, Rosti G et al (2005) ABL mutations in late chronic phase chronic myeloid leukemia patients with upfront cytogenetic resistance to imatinib are associated with a greater likelihood of progression to blast crisis and shorter survival: a study by the GIMEMA Working Party on Chronic Myeloid Leukemia. J Clin Oncol 23:4100-4109

42. Thiele J, Kvasnicka HM, Schmitt-Graeff A et al (2005) Bone marrow changes in chronic myelogenous leukaemia after longterm treatment with the tyrosine kinase inhibitor STI571: an immunohistochemical study on 75 patients. Histopathology 46:540-550

43. Lugli A, Ebnoether M, Cogliatti SB et al (2005) Proposal of a morphologic bone marrow response score for imatinib mesylate treatment in chronic myelogenous leukemia. Hum Pathol 36:91100

44. Thiele J, Kvasnicka HM, Varus E, Ollig E, Schmitt-Graeff A, Staib P, Griesshammer M (2004) Megakaryocyte features and bcr/ abl translocation in chronic myeloid leukemia following imatinib 
mesylate (STI571) therapy - a fluorescence in-situ hybridization study. Leuk Lymphoma 45:1627-1631

45. Rosti G, Testoni N, Martinelli G, Baccarani M (2003) The cytogenetic response as a surrogate marker of survival. Semin Hematol 40:56-61

46. Cervantes F, Mauro M (2011) Practical management of patients with chronic myeloid leukemia. Cancer (in press)

47. Landstrom AP, Tefferi A (2006) Fluorescent in situ hybridization in the diagnosis, prognosis, and treatment monitoring of chronic myeloid leukemia. Leuk Lymphoma 47:397-402

48. Kantarjian H, Schiffer C, Jones D, Cortes J (2008) Monitoring the response and course of chronic myeloid leukemia in the modern era of BCR-ABL tyrosine kinase inhibitors: practical advice on the use and interpretation of monitoring methods. Blood 111:1774-1780

49. Baccarani M, Cortes J, Pane F et al (2009) Chronic myeloid leukemia: an update of concepts and management recommendations of European LeukemiaNet. J Clin Oncol 27:6041-6051

50. Baccarani M, Saglio G, Goldman J et al (2006) Evolving concepts in the management of chronic myeloid leukemia: recommendations from an expert panel on behalf of the European LeukemiaNet. Blood 108:1809-1820

51. Branford S, Cross NC, Hochhaus A, Radich J, Saglio G, Kaeda J, Goldman J, Hughes T (2006) Rationale for the recommendations for harmonizing current methodology for detecting BCR-ABL transcripts in patients with chronic myeloid leukaemia. Leukemia 20:1925-1930

52. Wang L, Pearson K, Ferguson JE, Clark RE (2003) The early molecular response to imatinib predicts cytogenetic and clinical outcome in chronic myeloid leukaemia. Br J Haematol 120:990 999

53. Mahon FX, Rea D, Guilhot J et al (2010) Discontinuation of imatinib in patients with chronic myeloid leukaemia who have maintained complete molecular remission for at least 2 years: the prospective, multicentre Stop Imatinib (STIM) trial. Lancet Oncol 11:1029-1035

54. Press RD, Love Z, Tronnes AA et al (2006) BCR-ABL mRNA levels at and after the time of a complete cytogenetic response (CCR) predict the duration of CCR in imatinib mesylate-treated patients with CML. Blood 107:4250-4256

55. Branford S, Rudzki Z, Parkinson I et al (2004) Real-time quantitative PCR analysis can be used as a primary screen to identify patients with CML treated with imatinib who have BCRABL kinase domain mutations. Blood 104:2926-2932

56. Cortes J, Talpaz M, O'Brien S et al (2005) Molecular responses in patients with chronic myelogenous leukemia in chronic phase treated with imatinib mesylate. Clin Cancer Res 11:3425-3432

57. Quintas-Cardama A, Cortes JE, O'Brien S, Ravandi F, Borthakur G, Liu D, Bleickardt E, Chen TT, Kantarjian HM (2009) Dasatinib early intervention after cytogenetic or hematologic resistance to imatinib in patients with chronic myeloid leukemia. Cancer 115:2912-2921

58. Jones D, Kamel-Reid S, Bahler D et al (2009) Laboratory practice guidelines for detecting and reporting BCR-ABL drug resistance mutations in chronic myelogenous leukemia and acute lymphoblastic leukemia: a report of the Association for Molecular Pathology. J Mol Diagn 11:4-11

59. Hughes T, Deininger M, Hochhaus A et al (2006) Monitoring CML patients responding to treatment with tyrosine kinase inhibitors: review and recommendations for harmonizing current methodology for detecting BCR-ABL transcripts and kinase domain mutations and for expressing results. Blood 108:28-37

60. O'Hare T, Eide CA, Deininger MW (2007) Bcr-Abl kinase domain mutations, drug resistance, and the road to a cure for chronic myeloid leukemia. Blood 110:2242-2249
61. Willis SG, Lange T, Demehri S et al (2005) High-sensitivity detection of BCR-ABL kinase domain mutations in imatinib-naive patients: correlation with clonal cytogenetic evolution but not response to therapy. Blood 106:2128-2137

62. Saikia T, Advani S, Dasgupta A, Ramakrishnan G, Nair C, Gladstone B, Kumar MS, Badrinath Y, Dhond S (1988) Characterisation of blast cells during blastic phase of chronic myeloid leukaemia by immunophenotyping - experience in 60 patients. Leuk Res 12:499-506

63. Cortes JE, Talpaz M, Kantarjian H (1996) Chronic myelogenous leukemia: a review. Am J Med 100:555-570

64. Khalidi HS, Brynes RK, Medeiros LJ, Chang KL, Slovak ML, Snyder DS, Arber DA (1998) The immunophenotype of blast transformation of chronic myelogenous leukemia: a high frequency of mixed lineage phenotype in "lymphoid" blasts and A comparison of morphologic, immunophenotypic, and molecular findings. Mod Pathol 11:1211-1221

65. Sokal JE, Baccarani M, Russo D, Tura S (1988) Staging and prognosis in chronic myelogenous leukemia. Semin Hematol 25:49-61

66. Griesshammer M, Heinze B, Hellmann A, Popp C, Anger B, Heil G, Bangerter M, Heimpel H (1996) Chronic myelogenous leukemia in blast crisis: retrospective analysis of prognostic factors in 90 patients. Ann Hematol 73:225-230

67. Kantarjian HM, Deisseroth A, Kurzrock R, Estrov Z, Talpaz M (1993) Chronic myelogenous leukemia: a concise update. Blood 82:691-703

68. O'Brien SG, Guilhot F, Larson RA et al (2003) Imatinib compared with interferon and low-dose cytarabine for newly diagnosed chronic-phase chronic myeloid leukemia. N Engl J Med 348:9941004

69. Morris EL, Dutcher JP (2005) Blastic phase of chronic myelogenous leukemia. Clin Adv Hematol Oncol 3:547-552

70. Bujassoum S, Rifkind J, Lipton JH (2004) Isolated central nervous system relapse in lymphoid blast crisis chronic myeloid leukemia and acute lymphoblastic leukemia in patients on imatinib therapy. Leuk Lymphoma 45:401-403

71. Bornhauser M, Jenke A, Freiberg-Richter J, Radke J, Schuler US, Mohr B, Ehninger G, Schleyer E (2004) CNS blast crisis of chronic myelogenous leukemia in a patient with a major cytogenetic response in bone marrow associated with low levels of imatinib mesylate and its $\mathrm{N}$-desmethylated metabolite in cerebral spinal fluid. Ann Hematol 83:401-402

72. Gribble SM, Sinclair PB, Grace C, Green AR, Nacheva EP (1999) Comparative analysis of G-banding, chromosome painting, locusspecific fluorescence in situ hybridization, and comparative genomic hybridization in chronic myeloid leukemia blast crisis. Cancer Genet Cytogenet 111:7-17

73. O'Dwyer ME, Mauro MJ, Blasdel C, Farnsworth M, Kurilik G, Hsieh YC, Mori M, Druker BJ (2004) Clonal evolution and lack of cytogenetic response are adverse prognostic factors for hematologic relapse of chronic phase CML patients treated with imatinib mesylate. Blood 103:451-455

74. Wang Y, Hopwood VL, Hu P, Lennon A, Osterberger J, Glassman A (2004) Determination of secondary chromosomal aberrations of chronic myelocytic leukemia. Cancer Genet Cytogenet 153:53-56

75. Johansson B, Fioretos T, Mitelman F (2002) Cytogenetic and molecular genetic evolution of chronic myeloid leukemia. Acta Haematol 107:76-94

76. Melo JV, Barnes DJ (2007) Chronic myeloid leukaemia as a model of disease evolution in human cancer. Nat Rev Cancer 7:441-453

77. Salloukh HF, Laneuville P (2000) Increase in mutant frequencies in mice expressing the BCR-ABL activated tyrosine kinase. Leukemia 14:1401-1404 
78. Calabretta B, Perrotti D (2004) The biology of CML blast crisis. Blood 103:4010-4022

79. Asimakopoulos FA, Shteper PJ, Krichevsky S, Fibach E, Polliack A, Rachmilewitz E, Ben-Neriah Y, Ben-Yehuda D (1999) ABL1 methylation is a distinct molecular event associated with clonal evolution of chronic myeloid leukemia. Blood 94:2452-2460

80. Lima L, Bernal-Mizrachi L, Saxe D et al (2011) Peripheral blood monitoring of chronic myeloid leukemia during treatment with imatinib, second-line agents, and beyond. Cancer 117:1245-1252

81. Terre C, Eclache V, Rousselot P et al (2004) Report of 34 patients with clonal chromosomal abnormalities in Philadelphia-negative cells during imatinib treatment of Philadelphia-positive chronic myeloid leukemia. Leukemia 18:1340-1346

82. Loriaux M, Deininger M (2004) Clonal cytogenetic abnormalities in Philadelphia chromosome negative cells in chronic myeloid leukemia patients treated with imatinib. Leuk Lymphoma 45:2197-2203
83. Jabbour E, Kantarjian HM, Abruzzo LV, O’Brien S, GarciaManero G, Verstovsek S, Shan J, Rios MB, Cortes J (2007) Chromosomal abnormalities in Philadelphia chromosome negative metaphases appearing during imatinib mesylate therapy in patients with newly diagnosed chronic myeloid leukemia in chronic phase. Blood 110:2991-2995

84. Bumm T, Muller C, Al-Ali HK et al (2003) Emergence of clonal cytogenetic abnormalities in $\mathrm{Ph}-$ cells in some CML patients in cytogenetic remission to imatinib but restoration of polyclonal hematopoiesis in the majority. Blood 101:1941-1949

85. McMullin MF, Humphreys M, Byrne J, Russell NH, Cuthbert RJ, O'Dwyer ME (2003) Chromosomal abnormalities in $\mathrm{Ph}-$ cells of patients on imatinib. Blood 102:2700-2701, author reply 2701

86. Deininger M (2008) Resistance and relapse with imatinib in CML: causes and consequences. J Natl Compr Canc Netw 6(Suppl 2): S11-S21 